\title{
Optimal Primary-Backup Protocols
}

\author{
Navin Budhiraja ${ }^{\star}$, Keith Marzullo*, Fred B. Schneider ${ }^{\star \star}$, Sam Toueg ${ }^{\star \star}$ \\ Department of Computer Science, Cornell University, Ithaca NY 14853, USA
}

\begin{abstract}
We give primary-backup protocols for various models of failure. These protocols are optimal with respect to degree of replication, failover time, and response time to client requests.
\end{abstract}

\section{Introduction}

One way to implement a fault-tolerant service is to employ multiple sites that fail independently. The state of the service is replicated and distributed among these sites, and updates are coordinated so that even when a subset of the sites fail, the service remains available.

A common approach to structuring such replicated services is to designate one site as the primary and all the others as backups. Clients make requests by sending messages only to the primary. If the primary fails, then a failover occurs and one of the backups takes over. This service architecture is commonly called the primary-backup or the primary-copy approach [1].

In [5] we give lower bounds for implementing primary-backup protocols under various models of failure. These lower bounds constrain the degree of replication, the time during which the service can be without a primary, and the amount of time it can take to respond to a client request. In this paper, we show that most of these lower bounds are tight by giving matching protocols.

Some of the protocols that we describe have surprising properties. In one case, the optimal protocol is one in which a non-faulty primary is forced to relinquish control to a backup that it knows to be faulty! However, the existence of such a scenario is not peculiar to our protocol. As shown in [5], relinquishing control to a faulty backup is indeed necessary to achieve optimal protocols in some failure models. Another surprise is that in some protocols that achieve optimal response time, the site that receives the request (i.e. the primary) is not the site that sends the response to the clients. We show that this anomaly is not idiosyncratic to our protocols-it is necessary for achieving optimal response time.

\footnotetext{
* Supported by Defense Advanced Research Projects Agency (DoD) under NASA Ames grant number NAG 2-593 and by grants from IBM and Siemens.

** Supported in part by the Office of Naval Research under contract No0014-91-J1219, the National Science Foundation under Grant No. CCR-8701103, DARPA/NSF Grant No. CCR-9014363, and by a grant from IBM Endicott Programming Laboratory.

** Supported in part by NSF grants CCR-8901780 and CCR-9102231 and by a grant from IBM Endicott Programming Laboratory.
} 
The rest of the paper is organized as follows. Section 2 gives a specification for primary-backup protocols, Sect. 3 discusses our system model, Sect. 4 summarizes the lower bounds from [5], and Sect. 5 summarizes our results. Sections 6 , 7 and 8 describe the protocols that achieve our lower bounds, and Sect. 9 describes a protocol in which the primary is forced to relinquish control to a faulty backup. We conclude in Sect. 10. Due to lack of space, the description of some of the protocols and all proofs are omitted from this paper. See [4] for a complete description and proofs.

\section{Specification of Primary-Backup Services}

Our results apply to any protocol that satisfies the following four properties, and many primary-backup protocols in the literature (e.g. $[1,2,3])$ do satisfy this characterization.

Pb1: There exists predicate Prmys on the state of each site $s$. At any time, there is at most one site $s$ whose state satisfies Prmy .

$\mathrm{Pb} 2$ : Each client $i$ maintains a site identity Dest $_{i}$ such that to make a request, client $i$ sends a message (only) to Dest . $_{\text {. }}$

For the next property, we model a communications network by assuming that client requests are enqueued in a message queue of a site.

$\mathrm{Pb} 3$ : If a client request arrives at a site that is not the primary, then that request is not enqueued (and is therefore not processed by the site).

A request sent to a primary-backup service can be lost if it is sent to a faulty primary. Periods during which requests are lost, however, are bounded by the time required for a backup to take over as the new primary. Such behavior is an instance of what we call bofo (bounded outage finitely often). We say that an outage occurs at time $t$ if some client makes a request at that time but does not receive a response ${ }^{1}$. A $(k, \Delta)$-bofo server is one for which all outages can be grouped into at most $k$ periods, each period having duration of at most $\Delta{ }^{2}$. The final property of the primary-backup protocols is that they implement a bofo-server (for some values of $k$ and $\Delta$ ).

$\mathrm{Pb} 4$ : There exist fixed and bounded values $k$ and $\Delta$ such that the service behaves like a single $(k, \Delta)$-bofo server.

Clearly, $\mathrm{Pb} 4$ can not be implemented if the number of failures is not bounded. In particular, if all sites fail, then no service can be provided and so the service is not $(k, \Delta)$ for any finite $k$ and $\Delta$.

\footnotetext{
${ }^{1}$ For simplicity, we assume in this paper that every request elicits a response.

2 Therefore, as well as being finite, the number of such periods of service outages can occur is also bounded (by $k$ ).
} 


\section{The Model}

Consider a system with $n_{\mathrm{s}}$ sites and $n_{c}$ clients. Site clocks are assumed to be perfectly synchronized with real time ${ }^{3}$. Clients and sites communicate through a completely connected, point-to-point, FIFO network. Furthermore, if processes (clients or sites) $p_{i}$ and $p_{j}$ are connected by a (nonfaulty) link, then we assume for some a priori known $\delta$, a message sent by $p_{i}$ to $p_{j}$ at time $t$ arrives at $p_{j}$ at some time $t^{\prime} \in(t . . t+\delta]$.

We assume that all clients are non-faulty and consider the following types of site and link failures: crash failures (faulty sites may halt prematurely; until they halt, they behave correctly) ${ }^{4}$, crash + link failures (faulty sites may crash or faulty links may lose messages), receive-omission failures (faulty sites may crash or omit to receive some messages), send-omission failures (faulty sites may crash or omit to send some messages), general-omission failures (faulty sites may fail by send-omission, receive-omission, or both). Note that link failures and the various types of omission failures are different only insofar as a message loss is attributed to a different component. Link failures are masked by adding redundant communication paths; omission failures are masked by adding redundant sites. As we will see, the lower bounds for the two cases are different.

Let $f$ be the maximum number of components that can be faulty (i.e. $f$ is the maximum number of faulty sites in the case of crash, send-omission, receiveomission and general-omission failures, whereas $f$ is the maximum number of faulty sites and links in the case of crash+link failures).

\section{Lower Bounds}

In Tab. 1, we repeat the lower bounds from [5] for the degree of replication, the blocking time and the failover time for the various kinds of failures. Informally, a protocol is $C$-blocking if in all failure-free runs, the time that elapses from the moment a site receives a request until a site sends the associated response is bounded by $C{ }^{5}$ Failover time is defined to be the longest duration (over all possible runs) for which there is no primary. However, the failover time bounds only hold for protocols that satisfy the following additional (and reasonable) property.

$\mathrm{Pb} 5$ : $\mathrm{A}$ correct site that is the primary remains so until there is a failure.

${ }^{3}$ The protocols can be extended to the more realistic model in which clocks are only approximately synchronized [7].

4 The lower bounds are also tight for fail-stop failures [10] except for the bound on failover time.

${ }^{5}$ We assume that it takes no time for a site to compute the response to a request. 
Table 1. Lower Bounds-Degree of Replication, Blocking Time and Failover Time

\begin{tabular}{|c|c|c|c|}
\hline Failure type & Replication & Blocking time $(C)$ & Failover Time \\
\hline Crash & $n_{\mathrm{s}}>f$ & 0 & $f \delta$ \\
\hline Crash+Link & $n_{\mathrm{s}}>f+1$ & 0 & $2 f \delta$ \\
\hline Send-Omission & $n_{\mathrm{s}}>f$ & $\begin{array}{c}\delta \text { if } f=1 \\
2 \delta \text { if } f>1\end{array}$ & $2 f \delta$ \\
\hline Receive-Omission & $n_{s}>\left\lfloor\frac{3 f}{2}\right\rfloor$ & $\begin{aligned} \delta \text { if } n_{s} & \leq 2 f \text { and } f=1 \\
2 \delta \text { if } n_{s} & \leq 2 f \text { and } f>1 \\
0 & \text { if } n_{s}>2 f\end{aligned}$ & $2 f \delta$ \\
\hline General-Omission & $n_{\mathrm{s}}>2 f$ & $\begin{array}{c}\delta \text { if } f=1 \\
2 \delta \text { if } f>1\end{array}$ & $2 f \delta$ \\
\hline
\end{tabular}

\section{Summary of Results}

We first present a primary-backup protocol schema that will be used to derive the protocols for all the failure models. This schema is based on the properties of two key primitives, broadcast and deliver, that sites use to exchange messages. We show that the schema satisfies $\mathrm{Pb} 1-\mathrm{Pb} 5$ by only using these properties independent of the particular failure model. Each failure model-crash, crash+link, send-omission, receive-omission and general-omission-is handled with a different implementation of broadcast and deliver, and in all but one case optimal protocols are constructed.

The protocols for crash and crash+link failures show that all the corresponding lower bounds are tight. The protocol for general-omission failures uses a translation technique similar to [8], and demonstrates that our lower bounds for general-omission failures are tight, except for the bound on blocking time when $f=1$. However, for this special case we have derived a different protocol (not described in this paper) having optimal blocking time. In all failure free runs of this protocol, the site that receives the request (i.e. the primary) is not the site that sends the response to the client. We show that this behavior is necessary in this paper.

We do not show the protocols for send-omission and receive-omission failures in this paper because they are similar to the protocol for general-omission failures. These protocols establish that the bounds for send-omission failures are tight. For receive-omission failures, the lower bound on blocking time when $n_{\mathrm{s}}>2 f$ and the lower bound on failover time are also tight. However, our protocol does not have optimal replication, as it requires $n_{\mathbf{s}}>2 f$ (rather than $\left.n_{\mathrm{s}}>\left\lfloor\frac{3 f}{2}\right\rfloor\right)$.

Finally, in [5] we proved that all receive-omission protocols having $\left\lfloor\frac{3 f}{2}\right\rfloor<$ $n_{\mathrm{s}} \leq 2 f$ necessarily exhibit a scenario in which a non-faulty primary is forced to relinquish control to a faulty backup. In Sect. 9, we describe such a protocol: it uses two sites and tolerates a single receive-omission failure. In addition, this 
protocol is $\delta$-blocking and so it demonstrates that our lower bound on blocking time is tight for $n_{\mathrm{s}} \leq 2 f$ and $f=1$. As in the protocol for general omission when $f=1$, it is the backup that sends responses to clients. This behavior is shown to be necessary for an important class of protocols.

\section{Protocols for the Clients and the $(k, \Delta)$-bofo server}

Property $\mathrm{Pb} 4$ requires that the primary-backup service behave like some $(k, \Delta)$ bofo server. Figure 1 gives such a canonical $(k, \Delta)$-bofo server (say $s$ ), and Fig. 2 gives the protocol for client $i$ interacting with $s$. As with any other bofo server, a client will not receive the response to a request if either the request to $s$ or the response from $s$ is lost.

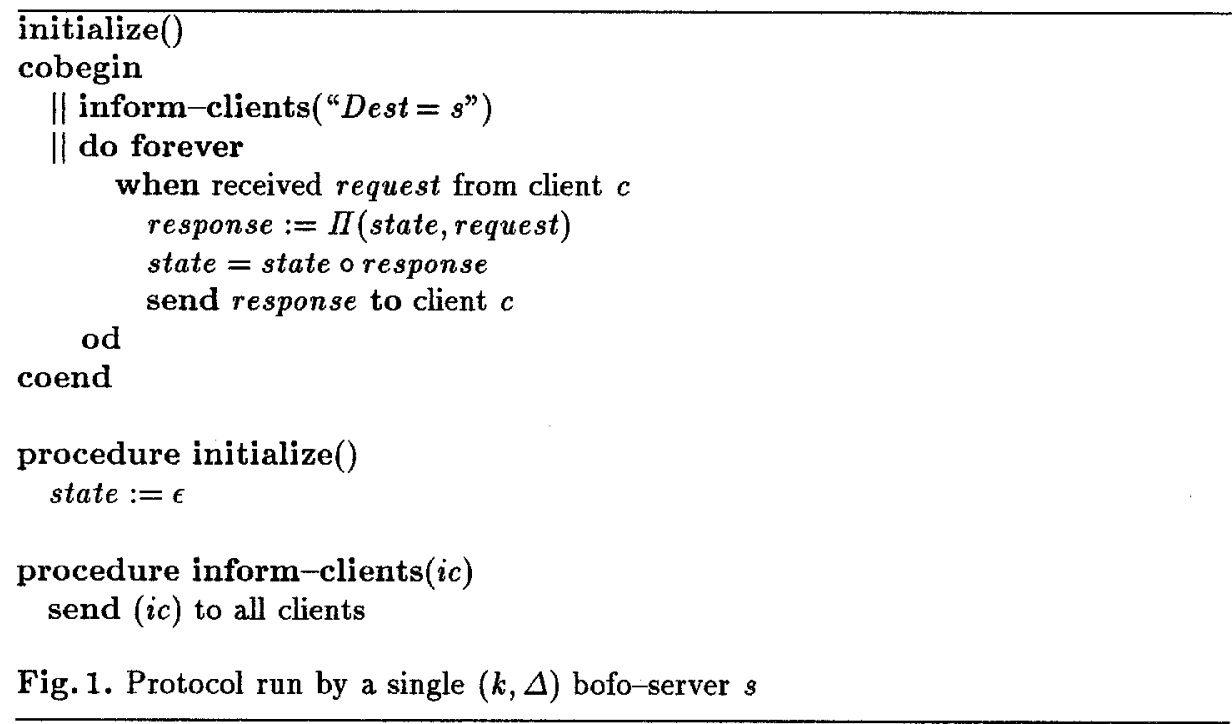

In Fig. 2, response-time corresponds to the amount of time the client has to wait in order to get the response from $s$, which is just the round trip message delay. The exact value for response-time depends on the failure model being assumed.

\section{The Primary-Backup Protocol Schema}

We first make the simplying assumption that the links between the clients and the sites are non-faulty and there are no omission failures between the clients and the sites (i.e. only the links between sites can be faulty for crash+link failures, 


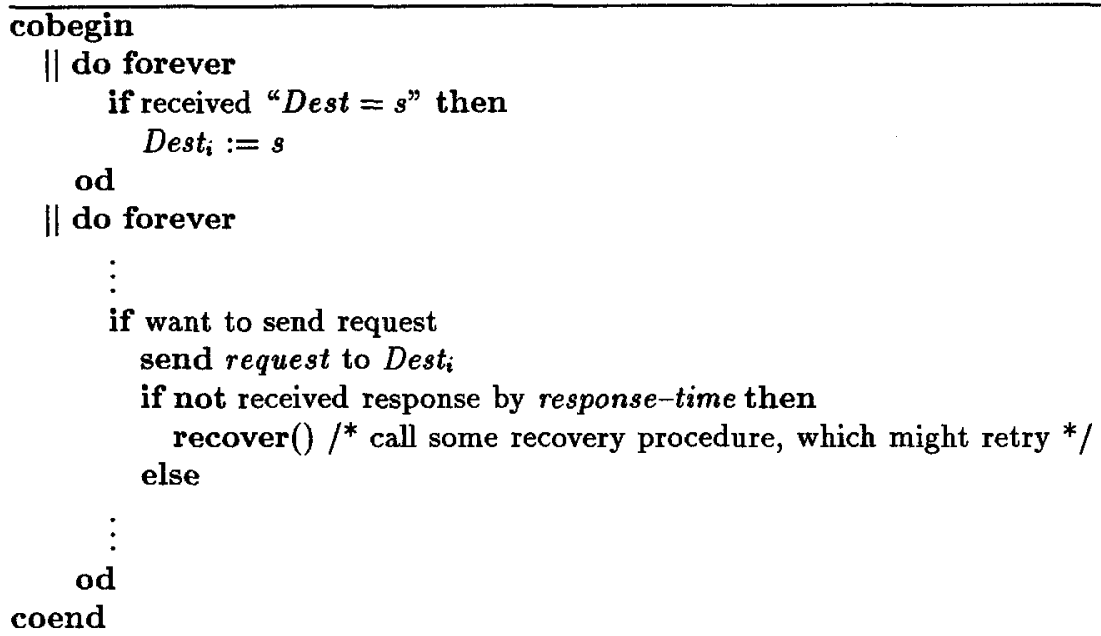

Fig. 2. Protocol run by client $i$ interacting with server $s$

and omission failures can occur only between sites for omission failures). We show in Sect. 7.1 how this assumption can be removed.

In order to emulate the server $s$ (and consequently satisfy property $\mathrm{Pb} 4$ ), our primary-backup protocol consists of $n_{\mathrm{s}}$ sites $\left\{s_{1}, \ldots, s_{n_{3}}\right\}$, each of which runs the protocol in Fig. 3. The protocol for the clients remains the same.

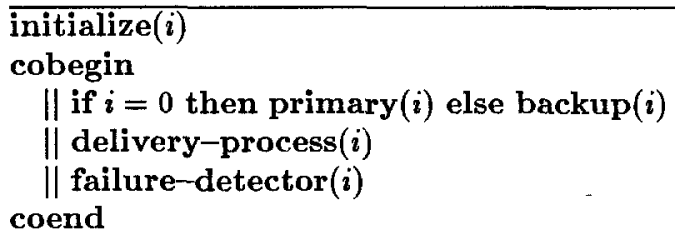

Fig. 3. Protocol run by site $s_{i}$ to emulate server $s$

The procedures primary and backup (shown in Fig. 4) are the same for all the failure models. On the other hand, the implementation of the procedures initialize, broadcast(used in Fig. 4), delivery-process and failure-detector change depending on the particular failure model. However, we ensure that these different implementations always satisfy a set of properties, called B1-B11 below. We extracted these properties in order to make our proofs modular. In particular we proved that, independent of the failure model, the protocol in Figs. 3 and 4 satisfies $\mathrm{Pb} 1-\mathrm{Pb} 5$, as long as the remaining procedures satisfy $\mathrm{B} 1-\mathrm{B} 11$. As a result, we could then prove $\mathrm{Pb} 1-\mathrm{Pb} 5$ for any other failure model 
by just ensuring that the implementation of broadcast, delivery-process and failure-detector for that failure model satisfied B1-B11.

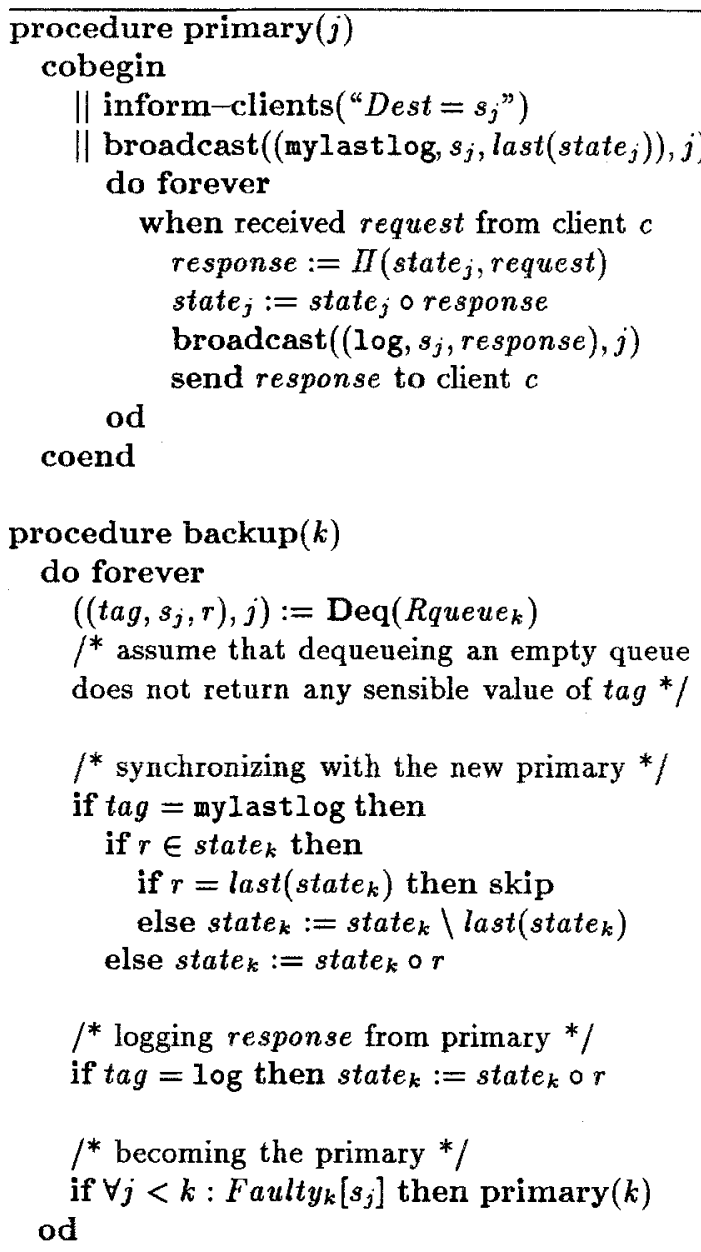

Fig. 4. The procedures primary and backup

We now give the properties $\mathrm{B} 1-\mathrm{B} 11$. In these properties, $d, C$ and $\tau$ are some constants whose values depend on the failure model. Intuitively, $d$ corresponds to the amount of time that can elapse from the time a message is broadcast to the time it is dequeued by the receiver, $C$ corresponds to the blocking time and $\tau$ corresponds to the interval between successive "I am alive" messages that sites send to each other (as we will see in the implementation of failure-detector). 
When we say that a site "halts", we mean that either the site has crashed or has stopped executing the protocol by executing a stop. The array of booleans Faulty $y_{k}$ indicates which servers $s_{k}$ believes has halted: Faulty $y_{k}\left[s_{j}\right]$ being true implies $s_{k}$ believes that $s_{j}$ has halted. Finally, we define a broadcast by a site to be successful if the site does not halt during the execution of broadcast.

The properties can be subdivided according to the procedures to which they relate:

Properties of broadcast and delivery-process:

B1: If $s_{j}$ initiates a broadcast $b^{\prime}$ after broadcast $b$, then no site dequeues $b^{\prime}$ before $b$.

B2: If $s_{j}$ initiates a broadcast $b$ at time $t$, then no site dequeues $b$ after time $t+d$.

B3: If $s_{j}$ initiates a broadcast at time $t$ and does not halt by time $t+C$, then the broadcast is successful. Furthermore, no broadcast takes longer than $C$ to complete.

Properties of failure-detector:

B4: If Fault $y_{j}\left[s_{k}\right]$ becomes true, then it continues to be true, unless $s_{j}$ halts.

B5: The value of Fault $y_{j}\left[s_{k}\right]$ can only change at time $t=l \tau+d$ for some integer $l \geq 0$.

B6: If Fault $y_{j}\left[s_{k}\right]=$ true at time $t$ then $s_{k}$ has halted by time $t$.

B7: If $s_{j}$ has not halted by time $t_{1}$, and $s_{i}, i<j$ has halted by time $t_{2}$ where $t_{1}=t_{2}+\tau+d$, then Fault $y_{j}\left[s_{i}\right]=$ true by time $t_{1}$.

Properties of broadcast and delivery-process interacting with failure-detector:

B8: No correct site halts in procedures initialize, broadcast, delivery-process or failure-detector.

B9: If $s_{j}$ initiates a successful broadcast at time $t$, then for all non-halted sites $s_{k}, k>j$, Fault $y_{k}\left[s_{j}\right]=$ false through time $\left[\frac{t}{\tau}\right\rceil \tau+d$.

B10: If $s_{j}$ initiates a successful broadcast $b$, then for every non-halted site $s_{k}$ : $\left(\right.$ Fault $y_{k}\left[s_{j}\right]=$ true $) \Rightarrow\left(s_{k}\right.$ has dequeued $\left.b\right)$.

B11: If $s_{j}$ initiates a broadcast $b$ at time $t$ and $s_{k}, k>j$ broadcasts $b^{\prime}$, then either no site dequeues $b$ after $b^{\prime}$, or Fault $y_{k}\left[s_{j}\right]=$ false through time $t+d$.

\subsection{Outline of the Proof of Correctness}

We now informally argue that the protocol in Figs. 3 and 4 satisfies $\mathrm{Pb} 1-\mathrm{Pb} 5$ as long as the procedures initialize, broadcast, delivery-process and failure-detector satisfy B1-B11.

Define: $\operatorname{Prm} y_{s_{j}}$ at time $t \equiv s_{j}$ has not halted by time $t$

$\wedge \forall k<j:$ Fault $y_{j}\left[s_{k}\right]=$ true at time $t$.

From the above definition, $\mathrm{Pb} 1$ can now be seen from $\mathrm{B} 6$ and the backup protocol in Fig. 4. Pb2 trivially follows from Fig. 2. Pb3 follows from Fig. 4 as 
no request is sent to a site $s_{j}$ before $s_{j}$ becornes the primary. Also, $\mathrm{Pb} 5$ holds (from B8 and Fig. 4) as a correct primary continues to be the primary. We now show $\mathrm{Pb} 4$.

In order to show $\mathrm{Pb} 4$, we need to show two things-the state of the new primary is consistent with the state of old primary; and all outages are bounded. We first show that the states are consistent.

Starting at the top of Fig. 4: when a site $s_{j}$ becomes the primary, it first informs the clients of its identity by calling inform-clients. For now, ignore the broadcast of (mylastlog, $s_{j},-$ ) by primary $s_{j}$.

Whenever $s_{j}$ gets a request from a client, it computes the response, changes state, broadcasts the $\log$ to the backups and sends the response back to the client. It can be seen from Fig. 4 that if primary $s_{j}$ sends a response $r$ to the client, then $s_{j}$ must have executed a successful broadcast of $\left(\log , s_{j}, r\right)$. This fact and properties $\mathrm{B} 1, \mathrm{~B} 2, \mathrm{~B} 9$ and $\mathrm{B} 10$ imply that $\left(\mathrm{log}, s_{j}, r\right)$ must also have been dequeued by any backup $s_{k}$ before $s_{k}$ becomes the primary. Thus, the state of $s_{k}$ will continue to be consistent with the state of $s_{j}$ iff the states were consistent when $s_{j}$ became the primary. We show this as follows.

Informally, the states of $s_{j}$ and $s_{k}$ could be inconsistent when $s_{j}$ becomes the primary for the following reason. Consider a scenario in which some primary $s_{i}$ crashes during the broadcast of $\left(\log , s_{i}, r\right)$ for some $r$. It is possible that $s_{k}$ received $\left(\log , s_{i}, r\right)$ and $s_{j}$ did not. As a result, the states of $s_{j}$ and $s_{k}$ now differ. It is for this reason that $s_{j}$ broadcasts (mylast $\left.l \circ g, s_{j}, r^{\prime}\right)$ where $r^{\prime}=\operatorname{last}\left(\right.$ state $_{j}$ ) on becoming the primary. On receiving this, $s_{k}$ sees that $r^{\prime} \neq \operatorname{last}\left(\right.$ state $\left._{k}\right)=$ $r$ and removes $r$ from its state. As a result, state $_{j}$ and state $_{k}$ become equal. Similarly, $s_{k}$ would add $r$ to its state had $s_{j}$, and not $s_{k}$, received $\left(\log , s_{i}, r\right)$.

In the scenario described in the last paragraph, response $r$ is never sent to the client (i.e. there is a service outage). We now show that such outages are bounded. $s_{i}$ did not send the response, and so by B3, must have halted by time $t$ (say). Now from B7 either $s_{i+1}$ halts or becomes the primary by time $t+\tau+\delta$. Since no correct site halts (by B8 and Fig 4), and the number of faulty sites are bounded by $f$, there eventually will be a time when there is a correct primary and no more outages occur.

From B3, the protocol $C$-blocking. Furthermore, it can be shown from B7, B8 and Fig. 4 that the failover time of the protocol is $f(d+\tau)$ for arbitrarily small and positive $\tau$.

However, the primary procedure in Fig. 4 does not work if there are message losses between the clients and the sites (due to link or omission failures). For example, a non-faulty primary might omit to receive all requests from a client due to a failure, violating Pb4. Similarly, inform-clients might omit to inform some of the clients. However, it is relatively easy to account for these failures when clients are non-faulty. Assume that there is an upper bound (say $G$ ) between any two requests from a client and that requests carry sequence numbers. If the primary does not receive any requests from a client during an interval of length $G$ or if the primary receives some request with a sequence number gap, then the primary halts. Similarly, the primary can detect that a response was 
lost by having clients acknowledge responses. If such an acknowledgement is not received, then again the primary halts. Properties $\mathrm{Pb} 1-\mathrm{Pb} 5$ can again be shown to be true if we make the above modification in Figs. 2 and 4.

\section{Implementation for the various Failure Models}

In this section, we show how to implement $\mathrm{B} 1-\mathrm{B} 11$ for the various failure models.

\subsection{Crash Failures}

The procedures implementing B1-B11 for crash failures are given in Fig. 5 . Whenever we say that a site "delivered $M$ ", we mean that the procedure deliver has been called with $M$. Enq adds an element to the head of a queue and Deq dequeues an element from the tail.

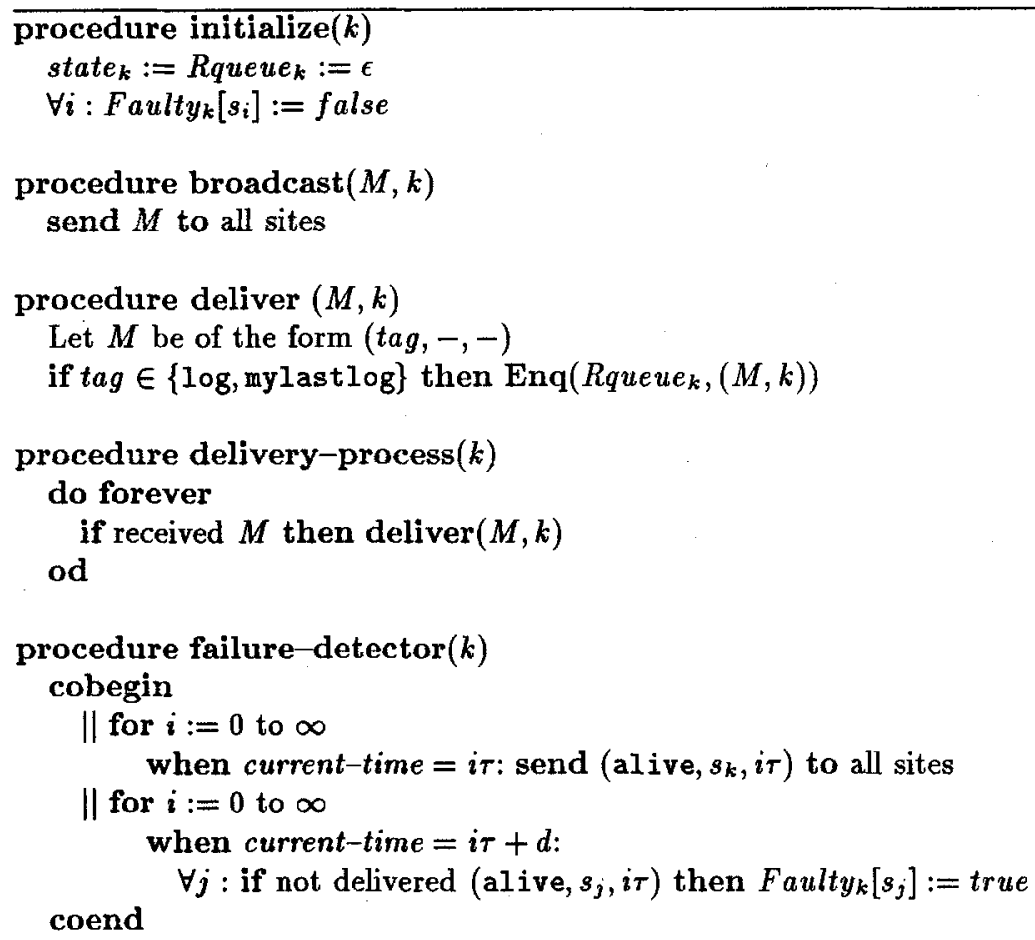

Fig. 5. Procedures for crash failures 
We now informally argue that B1-B11 hold for this implementation if $d=\delta$ and $C=0$. B1 holds as channels are FIFO and, B2 holds as $d=\delta$ and the maximum message delivery time is also $\delta$. B3, B4 and B5 can be seen trivially. $\mathrm{B} 6$ and $\mathrm{B} 7$ can be seen from failure-detector as there are no message losses and message delivery time is atmost $\delta$. B8 holds trivially. It can be shown that if $s_{j}$ halts at time $t$, then no site sets $F$ aulty $\left[s_{j}\right]$ to true before time $t+\delta$. B9, $\mathrm{B} 10$ and B11 now follow.

The procedures in Fig. 5 require $n_{s}>f$, and so the lower bound on the degree of replication is tight. Since $C=0$ and $d=\delta$, from Sect. 7.1, the lower bounds on blocking time and failover time are tight as well.

\subsection{Crash+Link Failures}

The procedures in Sect. 8.1 do not work if links can fail. For example, if $s_{j}$ sends a message to $s_{k}$ then the message might not reach $s_{k}$ due to a link failure (which will violate $\mathrm{B} 6$ and $\mathrm{B} 10$ ). We therefore replace the implementation in Fig. 5. with the one in Fig. 6, except that deliver is the same as before. For this implementation, $d=2 \delta$ and $C=0$. These procedures use fifo-broadcast and fifo-deliver in Fig. 7 which ensure that intermittent link failures become permanent failures: if $s_{j}$ fifo-broadcasts a message $m$ to $s_{k}$ and $s_{k}$ omits to fifo-deliver $m$, then $s_{k}$ will not fifo-deliver any subsequent message from $s_{j}$.

It can be shown (proof omitted) that this new implementation again satisfies B1-B11 if $n_{\mathrm{s}}>f+1$. Informally, this is true because of the following reason. Whenever $s_{j}$ initiates a broadcast of $M$ at time $t$, it sends $M$ to all sites, and the sites then relay $M$ to all other sites. Since $n_{\mathrm{s}}>f+1$, there is always at least one non-faulty path between any two non-crashed sites, where a path consists of zero or one intermediate sites. Therefore, if $s_{j}$ does not crash during the broadcast, then all non-crashed sites will deliver $M$ by time $t+2 \delta$. Furthermore B1 will be satisfied because of the FIFO properties of fifo-broadcast and fifo-deliver.

This crash+link protocol requires $n_{\mathbf{s}}>f+1$, is 0 -blocking (since $C=0$ ), and has a failover time of $f(2 \delta+\tau)$ (since $d=2 \delta$ ). Thus, all lower bounds for crash+link failures are tight.

\subsection{General-Omission Failures}

The implementation of the procedures for general-omission failures is given in Figs. 8 and 9, except delivery-process which is the same as Fig. 6 . Whenever, we say that a site "fifo-delivered $M$ ", we mean that the procedure fifo-deliver was called with $M$. These procedures were developed using a technique similar to [8] (although modified to work in our non-round-based model) which requires $n_{\mathrm{s}}>2 f$ and $d=2 \delta$. 


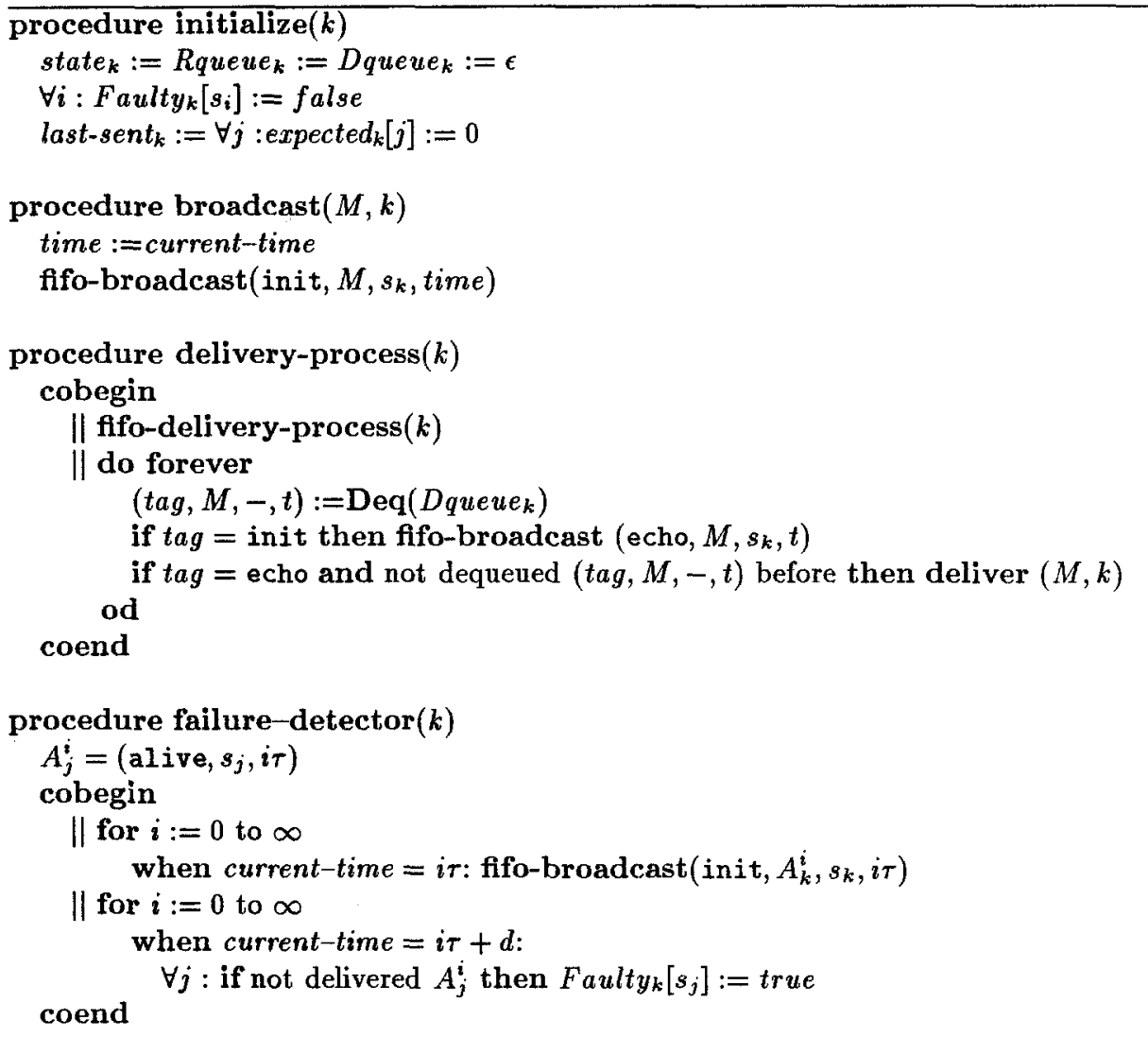

Fig. 6. Procedures for crash+link failures

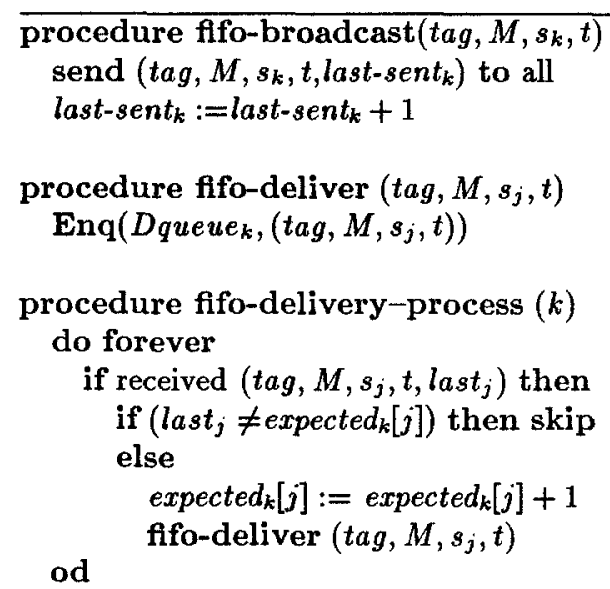

Fig. 7. Procedures for crash+link failures 


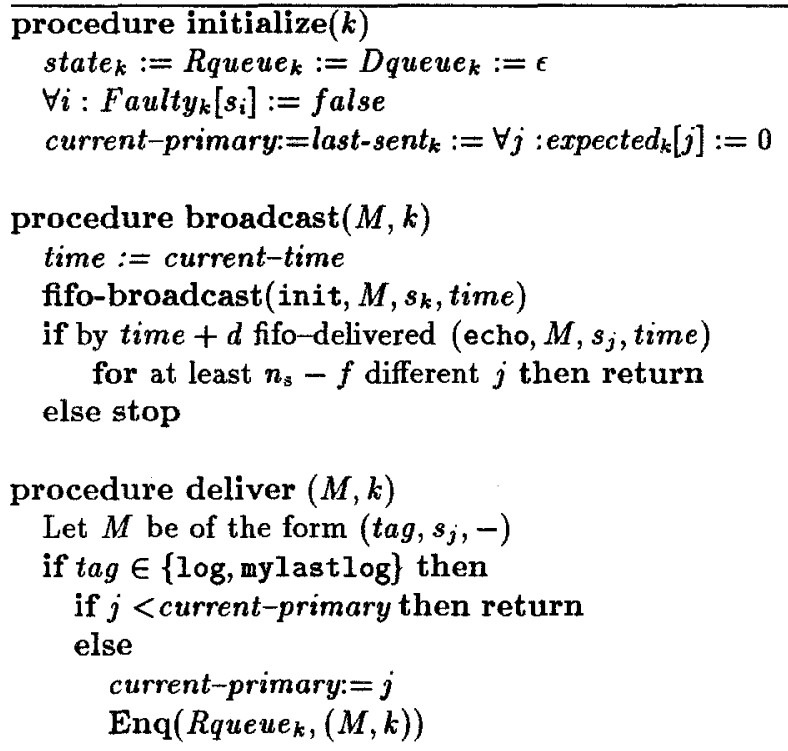

Fig. 8. Procedures for general-omission failures

We now briefly argue that these procedures satisfy B1-B11. The detailed proof is omitted from this paper. Had we used the implementation of broadcast in Fig. 6, B10 (in particular) would be violated because a faulty primary $s_{j}$ might omit to send the logs to the backups. Therefore, in Fig. $8, s_{j}$ stops in the broadcast of a response (say $r$ ) if less than $n_{\mathrm{s}}-f$ sites fifo-deliver and subsequently fifo-broadcast $r$. However, even if $s_{j}$ does not stop in the broadcast, a faulty (but non-crashed) site $s_{k}$ might still omit to deliver $r$, due to a receiveomission failure, and later become the primary were $s_{j}$ to fail. To prevent this, $s_{k}$ ensures (in procedure failure-detector) that it fifo-delivers some message (say $m^{\prime}$ ) from at least one of the above $n_{\mathrm{s}}-f$ sites that had earlier fifo-broadcast $r$. If $s_{k}$ does not receive such an $m^{\prime}$, then $s_{k}$ stops. Now, if $s_{k}$ omitted to fifo-deliver $r$, then by the properties of fifo-broadcast and fifo-deliver, $s_{k}$ cannot fifo-deliver $m^{\prime}$ and would stop (and, therefore, cannot become the primary). Property B6 is similarly satisfied by ensuring that sites detect their own failure to send or receive alive messages and therefore stop.

These procedures require $n_{\mathrm{s}}>2 f, d=2 \delta$ and $C=2 \delta$. Furthermore, we have developed a protocol for $f=1$ (omitted in this paper) that is $\delta$-blocking. Thus, we establish that all lower bounds for general-omission failures are tight.

As mentioned earlier, the $\delta$-blocking protocol for $f=1$ has scenarios in which the site that receives the request is not the site that responds to the clients. This is in fact necessary. Define a protocol to be "pass the buck" if in any failure-free run of the protocol, the site that receives a request is not the site that sends the corresponding response. 


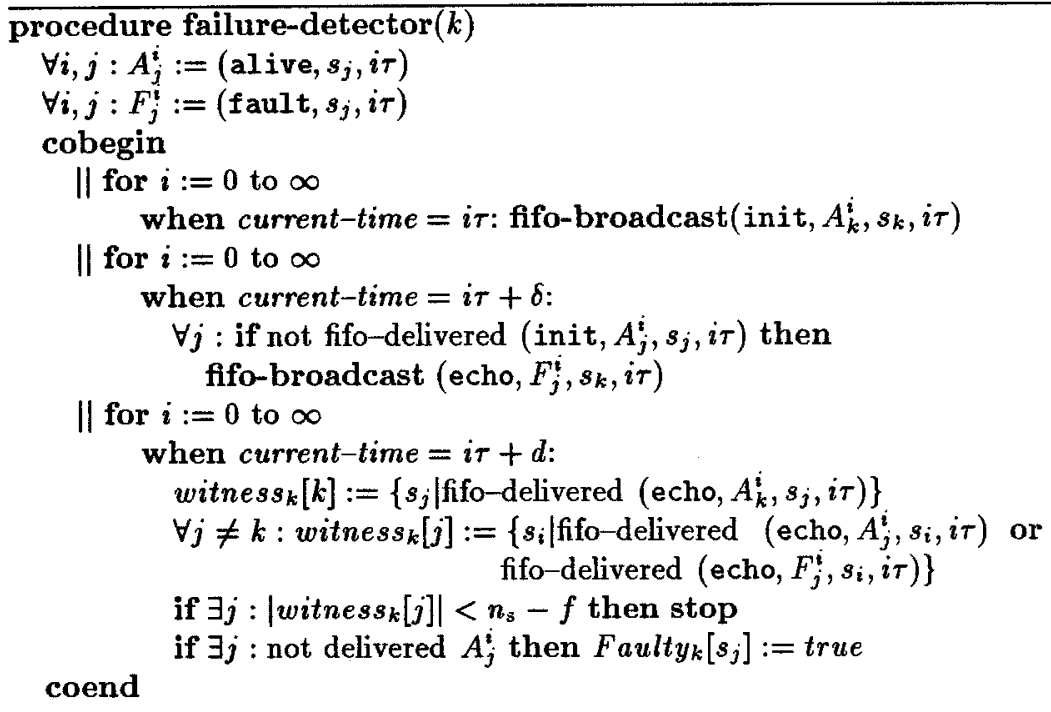

Fig. 9. Procedures for general-omission failures

Theorem 1. Any $C$-blocking protocol, where $C<2 \delta$, for send-omission failures is "pass the buck".

Proof. Omitted in this paper. See [4].

\subsection{Other Failure Models}

The implementations of the procedures for send-omission and receive-omission failures are similar to those for general-omission failures and so are omitted from this paper. For receive-omission failures, the lower bound on the degree of replication and the lower bound on blocking time when $n_{\mathrm{s}} \leq 2 f$ and $f>1$ are not tight. Finding optimal protocols remains an open problem. However, the lower bound on failover time for receive-omission failures, and all lower bounds for send-omission failures are tight.

\section{A Surprising Protocol}

We now describe a $\delta$-blocking protocol tolerating receive-omission failures for the special case of $n_{\mathrm{s}}=2$ and $f=1$. This protocol is complex, and so we omit the detailed description and only outline the protocol's operation here. This protocol shows that our lower bound on blocking time when $n_{\mathrm{s}} \leq 2 f$ and $f=1$ is tight. The protocol has the odd (yet necessary as shown in [5]) property that a non-faulty primary is forced to relinquish to a faulty backup. Furthermore, the protocol is "pass the buck". We, however, show that most $\delta$-blocking protocols tolerating receive omission failures have to be "pass the buck". 
Informally, let $\Gamma$ be the maximum time between any two successive client requests (possibly from different clients), and let $D$ be such that if some site $s$ becomes the primary at time $t_{0}$ and remains the primary through time $t \geq t_{0}+D$ when a client $i$ sends a request, then $D e s t_{i}=s$ at time $t$. We write $D<\Gamma$ to mean that $D$ is bounded and $\Gamma$ is either unbounded or bounded and greater than $D$. Then

Theorem 2. Any $C$-blocking protocol, where $C<2 \delta$, for receive-omission failures with $n_{\mathrm{s}} \leq 2 f$ and $D<\Gamma$ is "pass the buck".

Proof. Omitted from this paper.

Whether a protocol has to be "pass the buck" when the relation $D<\Gamma$ does not hold is an open question.

We now describe the protocol. There are two sites $s_{0}$ and $s_{1}$. They communicate with each other using fifo-broadcast and fifo-deliver shown in Fig. 7. Henceforth, when we say that a site sends a message to the other, we will mean that the message is sent with fifo-broadcast and other site receives it with fifo-deliver.

In a failure-free run of this protocol, since the backup responds to the client, the primary forwards any response to the backup (with a green tag as we see below) and the backup sends this response to the client. However, if there is a failure, then the primary responds to the clients. In this case, the primary forwards a response to the backup with a red tag. The backup does not forward a response to the client if the response has a red tag.

Let $s_{0}$ initially be the primary. Whenever $s_{0}$ receives a request from the client, it computes a response $r$, changes state, and sends (green, $r$ ) to $s_{1}$. Upon receiving this message, $s_{1}$ updates its state, acknowledges to $s_{0}$, and then sends $r$ to the client. Because it is the backup that responds to the client, the protocol is $\delta$-blocking. Site $s_{0}$ processes a new request only after receiving the acknowledgement from $s_{1}$ for the previous request. Finally, $s_{0}$ periodically sends alive messages to $s_{1}$, and $s_{1}$ acknowledges these messages.

Suppose that $s_{0}$ does not get $s_{1}$ 's acknowledgement for some message, say, (green, $r$ ) (the argument is similar if no acknowledgement is received for an alive message). There are three possibilities: (1) $s_{1}$ has crashed, (2) $s_{1}$ omitted to receive (green, $r$ ) and so did not send the acknowledgement, (3) $s_{0}$ omitted to receive the acknowledgement. $s_{0}$ now waits until it is supposed to send the next alive message. $s_{0}$ sends this alive message and waits for an acknowledgement. We now consider the above three cases separately.

Case 1: $s_{1}$ has crashed. As a result, $s_{0}$ does not receive the acknowledgement to the alive message. $s_{0}$ continues as the primary. From then on, whenever $s_{0}$ receives a request from the client, it computes the response $r$, sends (red, $r$ ) to $s_{1}$, and then sends the response back to the client. Also, $s_{0}$ continues to send alive messages. Since $s_{0}$ is correct, it can continue like this forever.

Case 2: $s_{1}$ is faulty and omitted to receive (green, $r$ ). By the property of fifobroadcast and fifo-deliver, $s_{1}$ will not receive the alive messages that $s_{0}$ sends. 
$s_{1}$ concludes that $s_{0}$ has crashed, sends (" $s_{1}$ is primary") to $s_{0}$ and becomes the primary. After that, it behaves like $s_{0}$ in case 1 above (including sending alive messages to $s_{0}$ ). Since $s_{0}$ is correct, it receives (" $s_{1}$ is primary") (as opposed to case 1) and so it becomes the backup. Also, since $s_{0}$ is correct it will not omit to receive (red,r) messages that $s_{1}$ sends and so $s_{0}$ keeps its state consistent with $s_{1}$. Subsequently, if $s_{0}$ stops receiving alive messages from $s_{1}$, then $s_{1}$ has crashed and $s_{0}$ becomes the primary once again.

Case 3: $s_{0}$ is faulty. Since $s_{1}$ is correct, it receives the alive message from $s_{0}$, sends the corresponding acknowledgement and remains the backup (as opposed to case 2). However, by the property of fifo-broadcast and fifo-receive, $s_{0}$ will not receive this acknowledge to the alive message (or the (" $s_{1}$ is primary") message), and so it behaves as in case 1 and continues as the primary. Similar to case $2, s_{1}$ receives all (red, $r$ ) messages that $s_{0}$ sends and so its state is consistent with $s_{0}$. Finally, $s_{1}$ becomes the primary if it stops receiving alive messages from $s_{0}$.

Case 2 in the protocol is the odd scenario in which the correct primary $s_{0}$ is being forced to relinquish to $s_{1}$, known to be faulty. However, this scenario is not something peculiar to our protocol. We showed in [5] that relinquishing to a faulty backup is necessary when $n_{\mathrm{s}} \leq 2 f$.

\section{Discussion}

In [5], we present lower bounds for primary-backup protocols which constrain the degree of replication, the failover time, and the amount of time it can take to respond to a client request. In this paper, we derive matching protocols and show that all except two of these lower bounds are tight. Furthermore, we show that in some cases the optimal response time can only be obtained if the site that receives the request is not site that sends the response to the clients.

We have attempted to give a characterization of primary-backup that is broad enough to include most synchronous protocols that are considered to be instances of the approach. There are protocols, however, that are incomparable to the class of protocols we analyze as these protocols were developed for an asynchronous system $[6,9]$. We are currently studying possible characterizations for a primarybackup protocol in an asynchronous system and expect to extend our results to this setting.

\section{References}

1. P.A. Alsberg and J.D. Day. A principle for resilient sharing of distributed resources. In Proceedings of the Second International Conference on Software Engineering, pages 627-644, October 1976.

2. J.F. Barlett. A nonstop kernel. In Proceedings of the Eighth ACM Symposium on Operating System Principles, SIGOPS Operating System Review, volume 15, pages 22-29, December 1981. 
3. Anupam Bhide, E.N. Elnozahy, and Stephen P. Morgan. A highly available network file server. In USENIX, pages 199-205, 1991.

4. Navin Budhiraja, Keith Marzullo, Fred B. Schneider, and Sam Toueg. Optimal primary-backup protocols. Technical report, Cornell University, Ithaca, N.Y., 1992. In preparation.

5. Navin Budhiraja, Keith Marzullo, Fred B. Schneider, and Sam Toueg. Primarybackup protocols: Lower bounds and optimal implementations. In Proceedings of the Third IFIP Working Conference on Dependable Computing for Critical Applications, 1992. To Appear.

6. Timothy Mann, Andy Hisgen, and Garret Swart. An algorithm for data replication. Technical Report 46, Digital Systems Research Center, 1989.

7. Gil Neiger and Sam Toueg. Substituting for real time and common knowledge in asynchronous distributed systems. In Sixth ACM Symposium on Principles of Distributed Computing, pages 281-293, Vancouver, Canada, August 1987. ACM SIGOPS-SIGACT.

8. Gil Neiger and Sam Toueg. Automatically increasing the fault-tolerance of distributed systems. In Proceedings of the Seventh ACM Symposium on Principles of Distributed Computing, pages 248-262, Toronto, Ontario, August 1988. ACM SIGOPS-SIGACT.

9. B. Oki and Barbara Liskov. Viewstamped replication: A new primary copy method to support highly available distributed systems. In Seventh ACM Symposium on Principles of Distributed Computing, pages 8-17, Toronto, Ontario, August 1988. ACM SIGOPS-SIGACT.

10. Richard D. Schlichting and Fred B. Schneider. Fail-stop processors: an approach to designing fault-tolerant computing systems. ACM Transactions on Computer Systems, 1(3):222-238, August 1983. 